\title{
Research on Bionic Polarization Direction Orientation Algorithm and Polarization Navigation Technology
}

\author{
Yanxin Jiang*, Zhaokui Meng, Gongliu Yang and Yajie Chen \\ School of Instrumententation Science and Opto-electronics Engineering, Beihang University, Beijing 100191, China \\ *Corresponding author
}

\begin{abstract}
Based on the standard atmospheric polarization model, the principle of bionic polarized light orientation is studied, and the formula of solar height angle and azimuth angle is given. Then the calculation method of polarization degree and polarization azimuth angle is studied. The method of calculating the heading angle when the carrier is tilted is deduced. The results show that if the horizontal angle of the carrier is known, the heading angle of the carrier can be calculated according to the polarization angle. Because the polarized light sensor can only give the heading information, the microelectromechanical system MEMS and the polarized light sensor combined navigation system are given. It is pointed out that the method of polarized light combined navigation can greatly improve the performance of the system and have a wide application field.
\end{abstract}

Keywords-sky polarized light; bionic navigation; directional algorithm; combined navigation

\section{INTRODUCTION}

The light emitted by the sun is natural light, and the natural light will polarize when it passes through the atmosphere. The direction and intensity of the polarized light are related to the orientation of the sun and the orientation of the observer[1-3]. The azimuth information of the polarized light can be used for orientation. Polarization navigation in a wide range by the impact of human factors is small, with strong interference and stability. The navigation information source is independent of GPS, inertial navigation, geomagnetic and other navigation methods, so it has important application value and development prospects. The polarized light sensor does not directly output the heading angle of the carrier. Currently, the polarized light navigation is considered to be horizontally mounted, thus simplifying the calculation process of the heading angle[5]. However, in practical applications, the polarized light sensor cannot always remain level. Polarization-based orientation method has the advantages of anti-interference and error not accumulating with time, and it is a completely autonomous navigation method. The combination of polarized light and SINS can effectively improve the navigation accuracy of the system, reduce the cost, but also will not add a greater burden on the carrier, with a wide range of applications.

\section{PRINCIPLE OF POLARIZED Light}

In order to describe the polarized light sensor orientation process, the coordinate systems involved are: navigation system (n series, north-east-ground), carrier system (b system, front-right-down), sensor coordinate system (m series, $\mathrm{X}$ and
Y-axis in the sensor-sensitive plane, Z-axis for the line of sight direction); related to the angle: three attitude angle (roll angle, pitch angle, heading angle, $\mathrm{r}, \theta, \psi$ )[7].

Select the north-east-ground geography coordinate system for the navigation system, according to the sun's azimuth $A_{s}$ and height angle $h_{s}$, you can get the direction of the sun in the navigation system under the projection:

$$
a_{s}^{n}=\left[\begin{array}{lll}
\cosh _{s} \cos _{s} & -\cosh _{s} \sin \mathrm{A}_{s} & -\sinh \\
s
\end{array}\right]^{T}
$$

The polarized light sensor outputs the angle between the reference direction of the sensor and the E-vector of the incident light $\phi$. According to the definition, The representation of the E-vector in the m-system is:

$$
a_{p}^{m}=\left[\begin{array}{lll}
\cos \phi & \sin \phi & 0
\end{array}\right]^{T}
$$

The direction of the line of sight of the polarized light sensor is expressed in the m system as:

$$
a_{l}^{m}=\left[\begin{array}{lll}
0 & 0 & 1
\end{array}\right]^{T}
$$

According to the Rayleigh scattering model, it can be seen that the maximum polarization direction (E-vector) of the incident light is perpendicular to the line of sight and the plane defined by the sun. There is:

$$
a_{p}^{m}=k a_{l}^{m} \times a_{s}^{m}
$$

In the formula, $a_{s}^{m}$ is the unit direction vector of the sun direction in the $\mathrm{m}$ system, $\mathrm{k}$ is a constant, so that the modal values on both sides are equal and can be obtained further:

$$
a_{p}^{m}=k a_{l}^{m} \times C_{b}^{m} C_{n}^{b} a_{s}^{n}
$$


In the formula, $a_{s}^{n}$ is for the observer and the sun connection vector in the $\mathrm{n}$ series projection, $C_{n}^{b}$ is for the navigation system to the carrier system direction cosine matrix, $C_{b}^{m}$ is for the carrier system to the sensor coordinate system direction cosine matrix, can be obtained by calibration.

To illustrate the principle of the azimuth angle of the polarized light sensor, it is assumed that the polarized light navigation sensor is placed horizontally and the observation direction is vertical upward. As shown in Fig. $1, \overline{O Z^{\prime}}$ is the direction is the observation direction, $\overline{O S}$ is the sun direction, $\mathrm{h}_{s}, \mathrm{~A}_{s}$ are the sun's height angle and the azimuth angle with respect to the north direction, respectively. The output polarization angle of the polarized light sensor $\angle B O E$ is the angle between the reference direction of the carrier and the Evector. According to the solar polarization model, it can be seen that the E-vector is perpendicular to the plane where the observation direction $\overline{O Z^{\prime}}$ and the direction $\overline{O S}$ of the sun are.

$$
\overline{O E}=k \overline{O S} \times \overline{O Z^{\prime}}
$$

where $\mathrm{k}$ is a real number, the purpose is to make both sides of the mold equal. With the horizontal placement of the polarized light navigation sensor, $\angle A O E=90^{\circ}$ there are. Considering the existence of a $180^{\circ}$ difference in the solution of the heading angle, we can get the carrier heading angle $\psi$ in the navigation system:

$$
\begin{aligned}
& \psi=-\left(\mathrm{A}_{s}-\phi-90^{\circ}\right) \text { or } \\
& \psi=-\left(\mathrm{A}_{s}-\phi+90^{\circ}\right)
\end{aligned}
$$



FIGURE I. BIONIC POLARIZED LIGHT ORIENTATION DIAGRAM

\section{Method of Calculation of Heading Angle}

In practical use, the polarized light sensor is usually installed in conjunction with the carrier system, the polarized light sensor will not always maintain the level. In the quasi- static condition, the estimation accuracy of the horizontal angle of the carrier is very high. The calculation method of the heading angle of the carrier is solved according to the polarization angle under the carrier level and the inclined condition respectively.

1) When $\mathrm{r}=\theta=0^{\circ}$, then

By the formula (5), as in

$$
\left[\begin{array}{c}
\cos \varphi \\
\sin \varphi \\
0
\end{array}\right]=k\left[\begin{array}{ccc}
-\sin \psi & \cos \psi & 0 \\
\cos \psi & \sin \psi & 0 \\
0 & 0 & 0
\end{array}\right]\left[\begin{array}{c}
\cos \mathrm{A}_{s} \\
-\sin \mathrm{A}_{s} \\
-\tanh
\end{array}\right] \cosh _{s}(8)
$$

In order to equalize the modulo at both ends of the equation, take $k= \pm \frac{1}{\cosh _{s}}$, there is

$$
\left[\begin{array}{c}
\cos \varphi \\
\sin \varphi \\
0
\end{array}\right]= \pm\left[\begin{array}{c}
-\sin \left(\psi+\mathrm{A}_{\mathrm{s}}\right) \\
\cos \left(\psi+\mathrm{A}_{\mathrm{s}}\right) \\
0
\end{array}\right]
$$

Further to get

$$
\begin{aligned}
& \psi=-\left(\mathrm{A}_{s}-\phi-90^{\circ}\right) \text { or } \\
& \psi=-\left(\mathrm{A}_{s}-\phi+90^{\circ}\right)
\end{aligned}
$$

The result is the same as equation (7).

Due to $k= \pm \frac{1}{\cosh _{s}}$ therefore, when the sun's height angle $h_{s}$ is close to $90^{\circ}$, the estimation error of the carrier heading angle will be very large.

2) $r=0^{\circ} \operatorname{or} \theta=0^{\circ}$

When $r=0^{\circ}$, By the formula (5) was

$$
\left[\begin{array}{c}
\cos \varphi \\
\sin \varphi \\
0
\end{array}\right]=k\left[\begin{array}{c}
-\sin \left(\psi+A_{s}\right) \\
\cos \theta \cos \left(\psi+A_{s}\right)+\sin \theta \tanh _{s} \\
0
\end{array}\right] \cosh _{s}
$$

Further:

$$
\frac{\cos \varphi}{\sin \varphi}=\frac{-\sin \left(\psi+A_{s}\right)}{\cos \theta \cos \left(\psi+A_{s}\right)+\sin \theta \tanh _{s}}
$$

Similarly, when $\theta=0^{\circ}$, then

$$
\frac{\cos \varphi}{\sin \varphi}=\frac{\sin \left(\psi+A_{s}\right) \operatorname{cosrcosh}_{s}+\operatorname{sinrsinh}_{s}}{-\cos \left(\psi+A_{s}\right) \cosh _{s}}
$$



$\psi$.

According to the above formula can solve the heading angle

3) $r \neq 0^{\circ}$ and $\theta \neq 0^{\circ}$

By the formula (5) we can get

$$
\begin{aligned}
& \frac{\cos \varphi}{\sin \varphi}= \\
& \frac{\operatorname{sinrsin} \theta \cos \left(\psi+A_{s}\right)-\operatorname{cosrsin}\left(\psi+A_{s}\right)-\operatorname{sinrcos} \theta \tanh _{s}}{\cos \theta \cos \left(\psi+A_{s}\right)+\sin \theta \tanh _{s}}
\end{aligned}
$$

The formula (13) degrades to the preceding results in special cases.

Make

$$
\begin{aligned}
& A=\cot \varphi \cos \theta-\sin r \sin \theta \\
& B=\cos r \\
& C=(\cot \varphi \sin \theta+\sin r \cos \theta) \tanh _{s}
\end{aligned}
$$

We can obtain:

$$
A \cos \left(\psi+A_{s}\right)+B \sin \left(\psi+A_{s}\right)+\mathrm{C}=0
$$

You can get it

$$
\sin \left(\psi+A_{s}+\beta\right)=\frac{-C}{\sqrt{A^{2}+B^{2}}}
$$

where: $\beta=\arctan \frac{A}{B}$

Solve the formula (16), we can get,

$$
\begin{aligned}
& \psi_{1}=\arcsin \left(\frac{-C}{\sqrt{A^{2}+B^{2}}}\right)-\beta-A_{s} o r \\
& \psi_{2}=\pi-\arcsin \left(\frac{-C}{\sqrt{A^{2}+B^{2}}}\right)-\beta-A_{s}
\end{aligned}
$$

Thus, a method of calculating the heading angle of the carrier according to the polarization angle can be obtained. When both horizontal angles are both $0^{\circ}, C=0$, There is $\arcsin \left(\frac{-C}{\sqrt{A^{2}+B^{2}}}\right)=0$,at this time $\psi_{1}$ and $\psi_{2}$ with a difference of $180^{\circ}$. However, when the two horizontal angles are not $0^{\circ}$, the ambiguity is not necessarily $180^{\circ}$, which is related to the rotation order defined by the three attitude angles. When the two horizontal angles are not very large, $\psi_{1}$ and $\psi_{2}$ with the difference in the $180^{\circ}$ or so.

\section{Polarization Azimuth Is CALCUlated Using A POLARIZED NAVIGATION SENSOR}

Based on the research results of insect eye complexes such as sand ants, polarized light sensors have been developed[7]. This sensor is modeled on the insect's visual nerve poles (POLOP units), using polarized opposing unit structures. The optical axes of the two polarizing plates are perpendicular to each other to form mutually orthogonal polarized light sensing channels; a corresponding interference filter is arranged above the polarizer so that the detection system is tuned to a fixed band; the logarithmic amplifier pair is from a quadrature Channel signal to the logarithmic op amp processing, to achieve similar POL-OP units of the polarization information processing functions.

In this way, the output of the sensitive unit is

$$
S(\varphi)=\mathrm{KI}\left[1+d \cos \left(2 \varphi-2 \varphi_{\max }\right)\right]
$$

where: I is the light intensity; $\mathrm{K}$ is the magnification; $\mathrm{d}$ is the degree of polarization of the polarized light; $\varphi$ is the direction of the current relative to the sun's meridian; $\varphi_{\max }$ is make the $S(\varphi)$ to achieve the maximum value of the direction.

In order to obtain the $\varphi$, the use of the general use of three polarized opposing units, respectively, and the body axis direction $0^{\circ}, 60^{\circ}$ and $120^{\circ}$. The output of the three polarized opposing units is

$$
P_{1}(\varphi)=\lg \frac{1+d \cos (2 \varphi)}{1-d \cos (2 \varphi)}
$$

$$
\begin{gathered}
P_{2}(\varphi)=\lg \frac{1+d \cos \left(2 \varphi-\frac{2 \pi}{3}\right)}{1-d \cos \left(2 \varphi-\frac{2 \pi}{3}\right)} \\
P_{3}(\varphi)=\lg \frac{1+d \cos \left(2 \varphi-\frac{4 \pi}{3}\right)}{1-d \cos \left(2 \varphi-\frac{4 \pi}{3}\right)}
\end{gathered}
$$

According to any of the two output signals can be resolved polarization azimuth $\varphi$. 


\section{Combinatorial Navigation TeChNOLOgy BASED ON POLARIZED LighT}

The essence of the integrated navigation is a kind of navigation technology which integrates and optimizes the information sent by each navigation sensor with the core signal processing and control platform as the center, and then synthesizes the navigation parameters synthetically[6]. The traditional inertial integrated navigation system has short-term accuracy and stability is better, navigation information is relatively complete, real-time strong, these are the advantages of traditional inertial navigation system. However, the traditional inertial navigation system cost is higher, the positioning error will accumulate over time, and cannot meet the navigation requirement of the long flight unmanned system when used alone. This requires us to seek a new way to overcome these shortcomings.

The polarized light navigation sensor can provide twodimensional compass information by checking the atmospheric polarization mode, providing navigation information to the navigation system[8]. In addition, polarized light biomedical navigation system has the advantages of small volume, high precision, good sensitivity, error does not accumulate with time, and cannot rely on external signal, with anti-jamming and so on. But it also has drawbacks, that is, polarized light bionic navigation system can only provide a direction of attitude, but cannot provide other gesture information[9]. Therefore, we want to combine the traditional inertial navigation system and polarized light sensor together to overcome the shortcomings of each other, learn from their own advantages, combined into a long can meet the needs of long time, the error does not accumulate with time inertial navigation system. Micro inertial device MEMS as a low-precision inertial navigation, with low cost, small size, but its accuracy there is no way to achieve long navigation when the autonomous navigation, so we according to MEMS and polarized light sensor advantages and disadvantages to be used polarized light sensor And its combination constitutes a polarized light / micro inertial integrated navigation system to meet the needs of autonomous navigation. In addition, if this micro-inertial integrated navigation system is used underwater, the target object in the water cannot be absolutely static, cannot compensate for the zero, so the introduction of the meter to provide speed constraints, the establishment of such a combination of ways to achieve navigation the goal of.

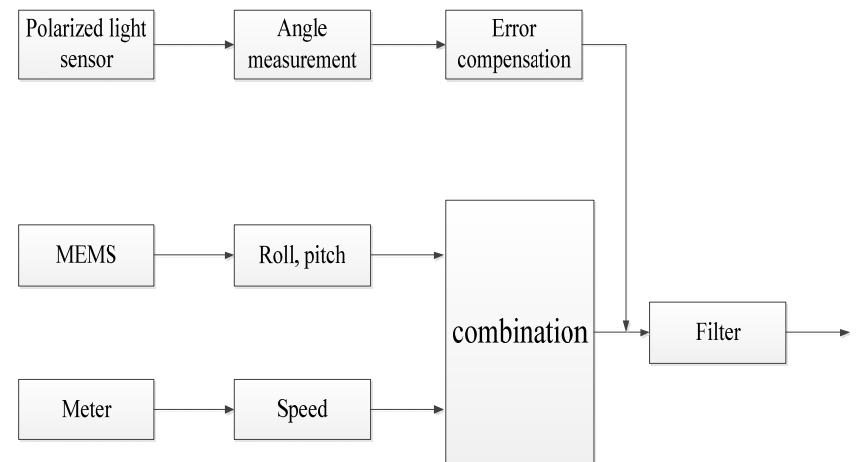

FIGURE II. POLARIZED LIGHT / MEMS INTEGRATED NAVIGATION SYSTEM

\section{CONCLUSION}

Based on the phenomenon of some polar migratory birds, sand ants and other insects using atmospheric polarized light navigation, this paper studies the principle of polarized light in the standard atmospheric polarization mode, deduces the method of calculating the heading angle when the carrier is tilted, The reason for the ambiguity exists when the heading angle is solved. The use of polarized light orientation alone can only be used for two-dimensional navigation and orientation. To achieve three-dimensional navigation, you can combine with SINS, the meter, etc. This combination will not bring too much burden on the carrier, that can achieve three-dimensional navigation. With the development of semiconductor microfabrication technology, micro-electromechanical systems (MEMS) have been developing rapidly[10]. The same is based on MEMS technology, micro-mechanical gyroscope and accelerometer is to practical, its long-term accuracy is not high weakness can be used to make up the observation of polarized light. And micro-electromechanical technology will be combined with the promotion of polarized light navigation technology miniaturization, practical one of the only way.

\section{ACKNOWLEDGMENT}

This research was financially supported by the National Science Foundation.

\section{REFERENCES}

[1] LABHART T, PETZOLD J, HELBLING H. Spatial Integration in Polarization-Sensitive Interneurones of Crickets: A Survey of Evidence, Mechanisms and Benefits[J]. The Jounal of Experimental Biology,2002,20(4):2423-2430.

[2] Cochran W W, Mouritsen H ,Wikelski M. Migrating songbirds recalibrate their magnetic compass daily from twilight cues [J].Science,2004,304:405-408.

[3] Muheim R,Philips J B,Akesson S. Polarized light cues underlie compass calibration in migratory songbirds[J].Science, 2006,313:837-839.

[4] Franz Matthias O, Mallot Hanspeter A. Biomimetic Robot Nacigation[J].Robotics and Autonomous Systems,2000,30(1):133-153.

[5] Lu Hongqian ,Yin Hang, Huang Xian-lin, Polarizedlight/geomagnetism/GPS/SINS integrated navigation [J]. Journal of Astronautics,2007,28(4):897-902.

[6] Chu Jin-kui, Wang Hong-qing, Rong Cheng-gong, et al. The performance test of a novel polarization sensor for navigation system [J]. Journal of Astronautice,2011,32(3):489-494.

[7] Wang Yu-jie, Hu Xiao-ping, Lian Jun-xiang, et al. Bionic Polarization Direction Finding Algorithm and Error Analysis[J]. Journal of Astronautice,2015,02:1000-1328.

[8] Chu J, Zhao K,Wang T, et al. Research on a Novel Polarization Sensor for Navigation: proceedings of the Proceedings of the 2007 International Conference on Information Acquisition, Jeju City, Korea,2007[C].

[9] Wang Y, Hu X, Zhang L, et al. An Evaluation of Skylight Polarization measurements[J].Sensors,2014,14(8):14916-14931.

[10] Xian Z, Hu X, Lian J,et al. A Novel Angle Computation and Calibration Algorithm of Bio-Inspired Sky-Light Polarization Navigation Sensor[J].Sensors,2014,14(9):17068-17088. 